\title{
Efficient beam shaping of linear, high-power diode lasers by use of micro-optics
}

\author{
Andreas Schilling, Hans Peter Herzig, Laurent Stauffer, Urs Vokinger, and \\ Markus Rossi
}

\begin{abstract}
We have designed, fabricated, and characterized a micro-optical beam-shaping device that is intended to optimize the coupling of an incoherent, linearly extended high-power diode laser into a multimode fiber. The device uses two aligned diffractive optical elements (DOEs) in combination with conventional optics. With a first prototype, we achieved an overall efficiency of $28 \%$. Straightforward improvements, such as antireflective coatings and the use of gray-tone elements, are expected to lead to an efficiency of approximately 50\%. The device is compact, and its fabrication is suited for mass production at low cost. This micro-optical device, used in a range-finder measurement system, will extend the measurement range. In addition to the direct laser writing technique, which was used for fabrication of the DOEs of the prototype, we applied two other technologies for the fabrication of the micro-optical elements and compared their performance. The technologies were multiple-projection photolithography in combination with reactive-ion etching in fused silica and high-energy beam-sensitive glass gray-tone lithography in photoresist. We found that refractive-type elements (gray tone) yield better efficiency for large deflection angles, whereas diffractive elements (multilevel or laser written) give intrinsically accurate deflection angles. (C) 2001 Optical Society of America
\end{abstract}

OCIS codes: $050.0050,050.1970,220.0220$.

\section{Introduction}

High-power, pulsed diode lasers are used in rangefinder systems for distance measurements. For these range finders the achievable measurement range depends directly on the available laser output power. In turn, the available laser output power per unit length is limited by the damage threshold of the material. Therefore, more output power results in a larger active region, and the geometrical shape of the emitting surface is typically a line. Efficient collimation of such a partially coherent, linearly extended light source with large divergence angles is a non-

When this study was performed, the A. Schilling (andreas.schilling@kinegram.com) and H. P. Herzig were with the Institute of Microtechnology, University of Neuchâtel, Rue A.-L. Breguet 2, CH-2000 Neuchâtel, Switzerland; L. Stauffer and U. Vokinger were with Leica Geosystems AG, Ch-9435 Heerbrugg, Switzerland; and M. Rossi was with the Centre Suisse d'Electronique et Microtechnique, Badenerstrasse 569, CH-8048 Zürich, Switzerland. M. Rossi is now with Heptagon, Zürich Office, Badenerstrasse 569, CH-8048 Zürich, Switzerland.

Received 17 November 2000; revised manuscript received 27 June 2001.

0003-6935/01/325852-08\$15.00/0

(C) 2001 Optical Society of America trivial problem whose solution is difficult to achieve with conventional optics.

We designed, fabricated, and characterized a micro-optical device with two aligned diffractive or refractive optical elements in combination with conventional optics. ${ }^{1}$ Our goal was to achieve high coupling efficiency of such a linear diode laser with a multimode fiber by use of a compact and low-cost optical system that is suited for mass fabrication. The diode laser was coupled into a multimode fiber to simplify quantification of its performance. In the final range-finder application the transformed light distribution was imaged onto the distant object. Leger and Goltsos ${ }^{2}$ used a similar concept to convert a mutually incoherent linear diode-laser array into a two-dimensional source with maximum radiance and symmetrized shape and divergence. Their system was designed to optimize end pumping of solid-state lasers.

\section{Working Principle}

Although the diode laser is fully coherent perpendicular to the active region, its spatial coherence extends to only a fraction of the total length in the parallel direction. Therefore a coherent beam-shaping element, a so-called random phase plate, for example, one designed by an iterative Fourier algorithm ${ }^{3,4}$ or a 

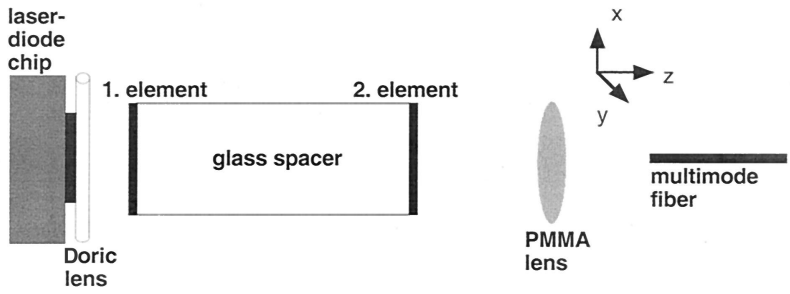

Fig. 1. Schematic of the optical setup: laser-diode chip (active region, $1 \mu \mathrm{m} \times 350 \mu \mathrm{m}$ ), combined DOE, additional poly(methyl methacrylate) (PMMA) imaging lens, and optical multimode fiber $(\phi=100 \mu \mathrm{m} ; \mathrm{NA}=0.2)$.

coherent addition with a fan-in element, ${ }^{5}$ is not appropriate for addressing the beam-shaping problem that is described here. The special coherence properties of the source necessitate an incoherent beamshaping method.

The main characteristics of a linear diode laser are the emitting wavelength $\lambda$, the lateral extensions of the active regions $d_{\perp}$ and $d_{\|}$, and the numerical apertures (NAs) $\mathrm{NA}_{\perp}=\sin \left(\theta_{\perp}\right)$ and $\mathrm{NA}_{\|}=\sin \left(\theta_{\|}\right)$ that are perpendicular and parallel, respectively. $\theta_{\perp}$ and $\theta_{\|}$are the corresponding divergence half-angles. The parallel direction refers to the $x$ axis and the perpendicular direction to the $y$ axis, as shown in Figs. 1 and 2. The diode laser that we used had a wavelength of $\lambda=850 \mathrm{~nm}$, lateral extensions of $d_{\perp}=$ $1 \mu \mathrm{m}$ and $d_{\|}=350 \mu \mathrm{m}$, and measured $\mathrm{NA}_{\perp}$ of 0.51 and $\mathrm{NA}_{\|}$of 0.12 . The one-dimensional spacebandwidth product (SBP) of a light source is defined as $^{6}$

$$
\mathrm{SBP}=\left(\frac{d}{2 \lambda}\right) \mathrm{NA}
$$

where $d$ is the lateral dimension. For a diffractionlimited Gaussian beam the SBP is ${ }^{7}$

$$
\mathrm{SBP}=1 / \pi
$$

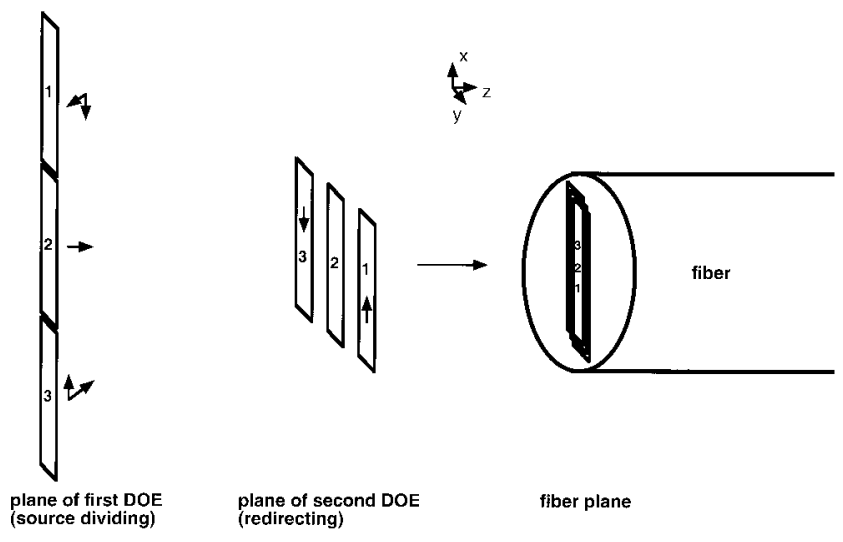

Fig. 2. Schematic working principle of the beam-shaping device (the Doric and the PMMA lenses are omitted for clarity). The parts of the source are denoted 1-3. The arrows mark the prism functions in the planes of the two DOEs.
The SBP of the high-power diode laser is therefore practically diffraction limited in the direction perpendicular to the active region and is typically a factor of 50 larger than for the diffraction-limited case in the parallel direction. The main problem for the beam shaping of the linear, edge-emitting diode laser lies in the fact that with conventional optics the SBP in one direction stays constant at best. Only the ratio between the NA and $d$ can be changed. The basic principle of our beam-shaping device is to symmetrize the lateral dimensions and the NA of the emitting source such that $\mathrm{SBP}_{\perp}=\mathrm{SBP}_{\|}$, with $\mathrm{SBP}_{\perp} \cdot \mathrm{SBP}_{\|}=$constant. For that purpose the line source is divided into three equal parts and redistributed on the fiber with the desired values for the NA and the lateral extensions. Consequently, we increase the coupling efficiency into the fiber and correspondingly enlarge the measurement range for range finders because of the increased intensity on the target.

Within the wave-propagation model, which was used for the theoretical description of the system, we modeled the linear diode laser as an incoherent superposition of 75 independent Gaussian sources with beam waists that corresponded to the measured divergence angles. Division of the source is achieved with the first micro-optical element. Ideally this division should be made directly in the source plane, but, because the fast-axis collimation has to be done first, the various mutually incoherent Gaussian beams begin to overlap in the direction parallel to the emitting edge. This overlap causes a small efficiency loss, which is approximately proportional to the region of overlap between neighboring incoherent Gaussian beams and therefore is approximately proportional to the distance between the active region of the source and the first diffractive optical element (DOE).

\section{Optical Setup}

The complete optical setup is displayed schematically in Fig. 1. The fast and the slow NAs of the diode laser were measured to be $\mathrm{NA}_{\perp}=0.51$ and $\mathrm{NA}_{\|}=$ 0.12 , respectively. A cylindrical gradient-index lens (Doric Lenses, Inc., Canada), which had a specified effective NA of 0.5 and a diameter of $60 \mu \mathrm{m}$, was mounted directly after the active region to collimate the fast axis. Inasmuch as the tolerances for mounting the gradient-index lens are extremely tight, we performed the alignment manually, using micrometer stages, by measuring the far-field intensity distribution and finally glued the lens onto the laser chip. The theoretical NA after the Doric lens was $\mathrm{NA}_{\perp}=0.0083$.

The beam-shaping device was then added. It was composed of two DOEs with a glass spacer between them. The first DOE, with a size of $370 \mu \mathrm{m} \times 370$ $\mu \mathrm{m}$, divides the line source into three equal parts along the parallel direction and directs them into three different directions. The working principle is shown schematically in Fig. 2 . In parts 1 and 3 of the first DOE, three optical functions are implemented: a perpendicular (along the $y$ axis) prism func- 


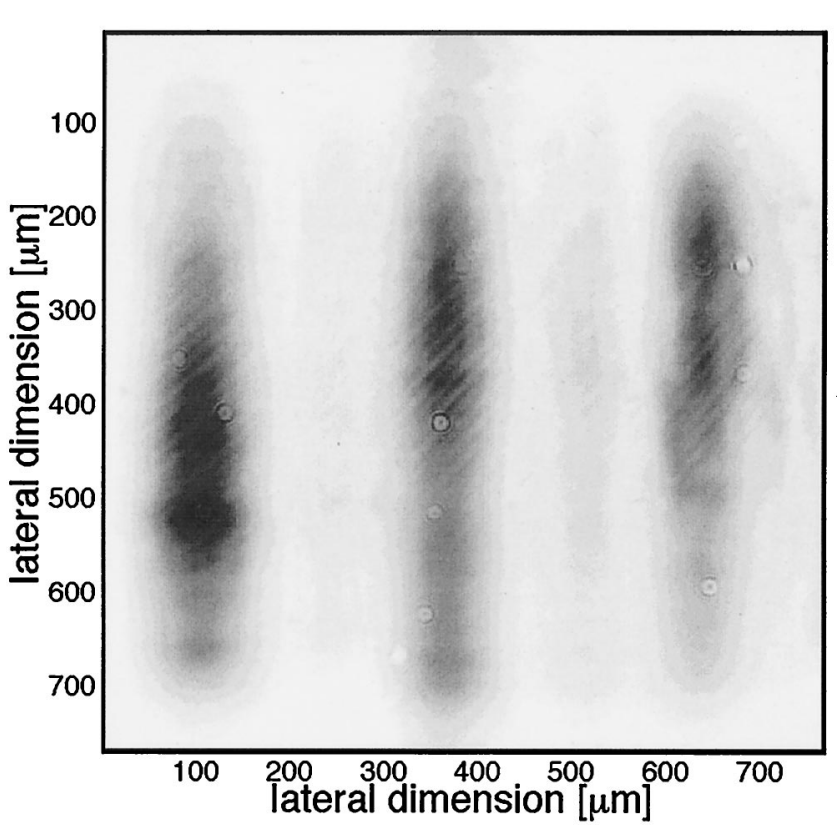

Fig. 3. Typical measured two-dimensional intensity distributions in the plane of the second DOE.

tion, a parallel (along the $x$ axis) prism function, and a focusing function parallel to the emitting edge of the diode laser. Central part 2 has only a focusing function, which collimates the beam parallel to the active region.

A typical measured intensity distribution in the plane of the second DOE is shown in Fig. 3. The second DOE, with a size of $800 \mu \mathrm{m} \times 800 \mu \mathrm{m}$, redirects parts 1 and 3 of the intensity distribution in the direction parallel to the emitting edge. The final conventional PMMA lens after the second DOE has two optical functions: It creates a reduced image of the transformed source at the fiber plane and at the same time serves to superpose the three parts of the
Table 1. Experimental Efficiencies of the Single Diffractive Elements ${ }^{a}$

\begin{tabular}{lc}
\hline \multicolumn{1}{c}{ Part of Element } & Measured Efficiency $(\%)$ \\
\hline First element, left & 70 \\
First element, center & 84 \\
First element, right & 68 \\
Second element, up & 79 \\
Second element, center & 98 \\
Second element, bottom & 81 \\
\hline
\end{tabular}

${ }^{a}$ Efficiencies are normalized with respect to transmitted intensity through an unstructured substrate.

source one upon the other. The multimode fiber has a diameter of $100 \mu \mathrm{m}$ and a NA of 0.2 .

\section{Results and Discussion}

The two DOEs were fabricated by direct laser writing 8,9 in photoresist with the laser writer at the Centre Suisse d'Electronique et Microtechnique, Zurich, Switzerland. Figures 4(a) and 4(b) show microscope pictures of the first and the second elements, respectively. Because the elements are quite small, a large number of elements can be written on one wafer. Subsequent replication and mounting procedures are also wafer compatible, so parallel fabrication technology can be used until the dicing of the combined DOEs is complete. Therefore fabrication, alignment, and mounting can be parts of a low-cost massfabrication process.

With focused light from a vertical-cavity surfaceemitting laser with a wavelength of $850 \mathrm{~nm}$ the diffraction efficiencies of the two DOEs were characterized before mounting. The measured efficiencies for the various sections of the two elements are summarized in Table 1 . The first element had an average efficiency of $74 \%$; the second element, of $87 \%$. We then aligned the two DOEs and a borofloat $600-\mu \mathrm{m}$-thick glass spacer and glued them together with UV-curable lens-bonding optical cement by us-
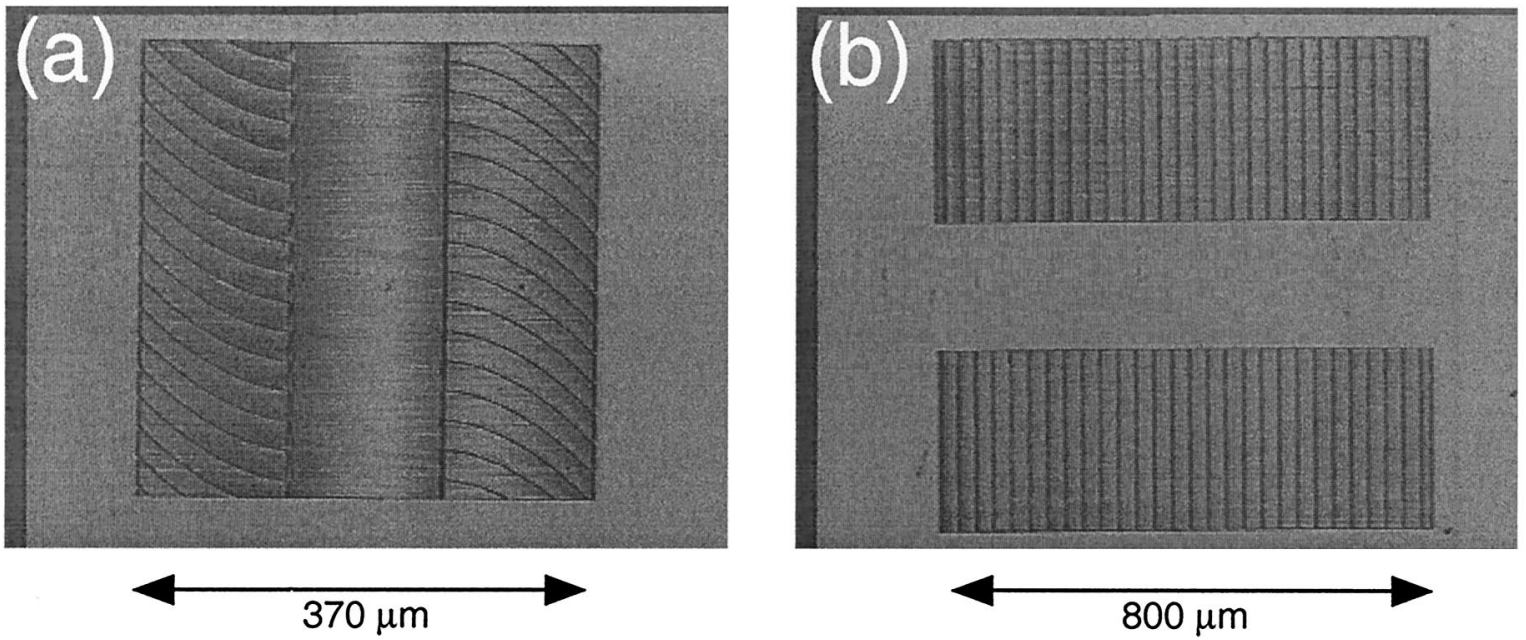

Fig. 4. Microscope pictures of the two DOEs: (a) the first element, close to the diode laser, (b) the second element. The elements were fabricated by direct laser writing in a photoresist. 

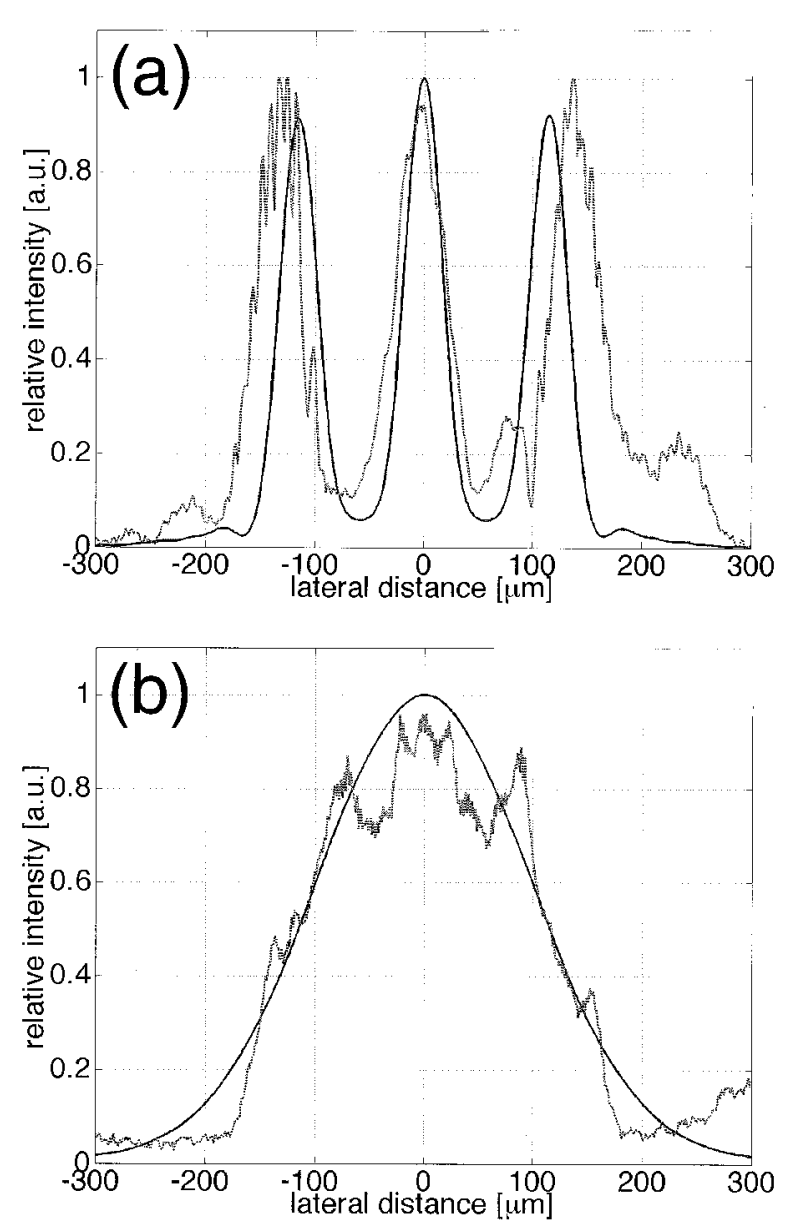

Fig. 5. Calculated and measured intensity distributions in the plane of the second DOE: (a) cross section parallel to the slow axis of the diode laser, (b) cross section parallel to the fast axis of the diode laser. Solid curves, theory; dashed curves, experiment.

ing a Karl Suess mask aligner, Model MA/BA6. The alignment marks were written by the laser writer at the same time as the DOEs. The alignment of the two DOEs with respect to each other had a tolerance of better than $5 \mu \mathrm{m}$. The total thickness of the combined DOE after mounting was $3 \mathrm{~mm}$.

For the theoretical optimization of the system design, we used the commercially available ray-tracing program Code V. The performance of the final structure was then analyzed quantitatively with a wave-propagation model that used different propagation methods, as described in detail in Ref. 7. Diffraction at apertures is fully taken into account in this model, and Fresnel losses at the optical surfaces can be considered approximately. For a comparison of the theoretical and the experimental intensity distributions, we used a wave-propagation model with which we modeled the linear diode laser by using an incoherent superposition of 75 independent Gaussian sources with beam waists that corresponded to the measured divergence angles.

Calculated and measured intensity cross sections in the plane of the second DOE and in the fiber plane are shown in Figs. 5 and 6, respectively. The agree-
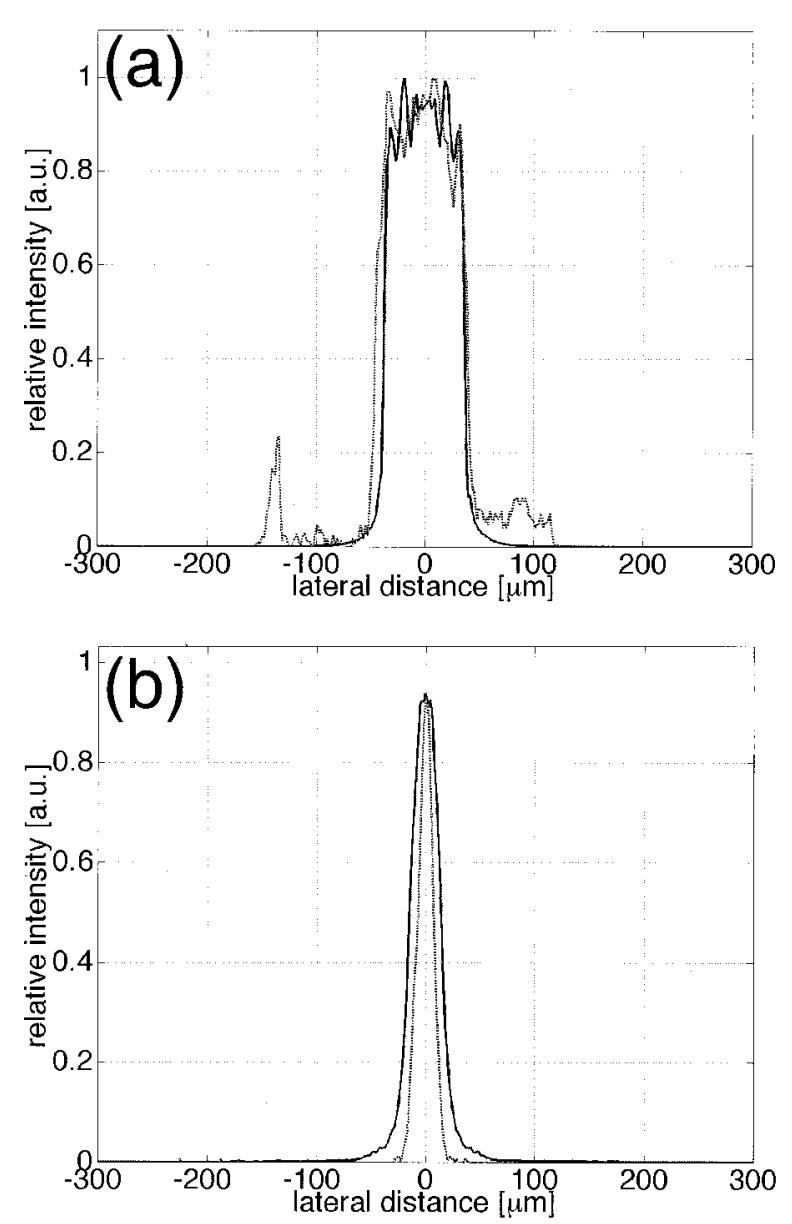

Fig. 6. Calculated and measured intensity distributions in the plane of the fiber: (a) cross section parallel to the slow axis of the diode laser, (b) cross section parallel to the fast axis of the diode laser. Solid curves, theory; dashed curves, experiment.

ment between the theoretical and the experimental intensity distributions is very satisfying. A possible cause of the observed small differences could be nonuniformity of the emitted intensity at the active region of the diode laser, and the cause of the possibly limited accuracy of the description of the source may be the incoherent superposition of Gaussian beams. The experimental nonuniformity of the source can also be seen in Fig. 3 .

We measured a value of approximately $80 \%$ for the collimating efficiency of the gradient-index lens. With the wave-propagation model and taking into account Fresnel losses, we obtained a value of approximately $88 \%$. For the theoretical value, a $4 \%$ loss originates from diffraction effects at the aperture, and another $8 \%$ is from Fresnel effects.

The overall efficiency of the beam-shaping device is given by the ratio of the power of the light coupled into the fiber to the power emitted by the laser. The overall efficiency that we obtained experimentally was $28 \%$; the theoretical value was $80 \%$, assuming ideal DOEs and no Fresnel losses. The difference between the theoretical and the experimental values has two main reasons: First, there are Fresnel 
Table 2. Experimental Efficiencies of the Laser-Written Elements and Estimated Optimization Potential Considering Antireflection Coatings and Micro-Optical Elements Fabricated by Gray-Tone Technology

\begin{tabular}{lcc}
\hline & \multicolumn{2}{c}{ Overall Efficiency (\%) } \\
\cline { 2 - 3 } \multicolumn{1}{c}{ Position } & $\begin{array}{c}\text { Optimization Potential } \\
\text { between Subsequent } \\
\text { Experimental } \\
\text { Efficiency }\end{array}$ & $\begin{array}{c}\text { Positions } \\
\text { Laser output }\end{array}$ \\
After gradient-index lens & 100 & 5 \\
After first DOE & 80 & 8 \\
After second DOE & 48 & 2 \\
Fiber & 38 & 7 \\
\hline
\end{tabular}

losses, which acount for approximately $14 \%$ of the total loss and can be eliminated by antireflection coatings on all optical surfaces. Second, the nonideal DOEs are responsible for an overall efficiency loss of approximately $27 \%$. The DOEs, especially the first element with smaller grating periods, can be replaced with refractive-type elements that have increased efficiency and are fabricated by high-energy beam-sensitive (HEBS) glass gray-tone technology. A comparison of technologies for the fabrication of the two micro-optical elements is made in Section 5 below. As shown below, an efficiency increase of approximately $10 \%$ for the first element is realistic and would yield an approximately 50\% overall efficiency for an optimized device including antireflection coatings. The reasons for the fundamental losses are mainly of two kinds: coupling losses at the collimation of the fast angle by the gradient-index lens, which amount to approximately $4 \%$ of the efficiency, and overlap losses of approximately $5 \%$ that originate from the fact that certain coherent source points hit two different parts of the first DOE. The second loss is limited by the minimum distance between the diode laser and the first micro-optical element, approximately $100 \mu \mathrm{m}$, and is determined mainly by the gradient-index lens with the smallest available diameter of $60 \mu \mathrm{m}$. The remaining $11 \%$ loss is due to diffraction effects at the apertures of the system. Table 2 summarizes the experimentally determined efficiencies and the estimated optimization potential.

Inasmuch as the NA of the impinging light at the fiber plane is important for the coupling efficiency, we measured the NA for the fast and the slow axes in the fiber plane and calculated the corresponding values by using the wave-propagation model. We obtained for the slow axis an experimental NA of 0.12 , compared with a theoretical NA value of 0.16 . For the fast axis, we obtained an experimental NA of 0.09 and a theoretical NA of 0.11. The experimental and the theoretical values for the fast axis agree reasonably well, whereas for the slow axis the experimental value is significantly smaller. A possible reason for the difference between the experimental and the theoretical values for the slow axis could lie in the simplified description of the diode laser as a superposition of incoherent Gaussian souces within the wave-propagation model.

\section{Comparison of Fabrication Technologies}

\section{A. Fabrication Methods}

Besides direct laser beam writing in photoresist, we used two other techniques for fabrication of the two micro-optical elements of the beam-shaping device: binary mask photolithography in combination with reactive-ion etching, yielding eight-level fused-silica elements, and HEBS glass gray-tone lithography in photoresist, yielding deep, continuous surface-relief structures. We compare the performance of the fabrication technologies with respect to the two microoptical elements that were used for the beam-shaping device and discuss the sources of loss.

Laser direct writing in photoresist 8,9 is an approach to obtaining continuous surface-relief profiles of diffractive or refractive optical elements with profile depths of as much as $30 \mu \mathrm{m}$. We used the laser writer at the Centre Suisse d'Electronique et Microtechnique, Zurich, which utilizes a focused $\mathrm{HeCd}$ laser beam $(\lambda=442 \mathrm{~nm})$ to expose a photoresist-coated substrate in a raster scan. We used a pixel size of $400 \mathrm{~nm}$ for the raster scan. Afterward, the photoresist was developed, resulting in a surface-relief structure. The direct laser writing technique is a flexible and fast method for obtaining prototypes without the need to generate masks, as in photolithographic methods.

Binary mask photolithography in combination with reactive-ion etching produces multilevel profiles. ${ }^{10}$ The substrate, fused silica, which is coated with a thin photoresist layer, is exposed through a binary chromium mask by use of a UV lamp. The chromium mask is normally fabricated by laser beam or electron beam writing, depending on the required resolution. After the development step a resist pattern remains, which is then transferred into the substrate in an etch step. A subsequent photolithographic step creates a refined resist pattern, which is then again transferred into the substrate, yielding a four-level surface profile. For eight- or sixteen-level surface profiles, correspondingly more aligned lithography and etch steps are necessary.

HEBS glass gray-tone technology uses a mask that has a continuous variation of transmission (gray levels). This variation is achieved because HEBS glass, ${ }^{11}$ which is fabricated by a silver-ion exchange process, ${ }^{12,13}$ when it is exposed to a high-energy electron beam suffers a reduction in silver ions, and the optical density of the material changes. The optical density increases with the electron dosage; typical values are $0-2.6$ for a wavelength of $\lambda=365 \mathrm{~nm}$. A major difficulty is that one has to work in the nonlinear regime of the photoresist response, unlike for binary mask photolithography. For the gray-tone technology, one needs to establish a calibration curve of resist height as a function of electron dosage by using a set of calibration structures. This calibration curve is afterward used to encode the profile of the designed surface-relief structure into an electron dose per pixel for the electron beam writing procedure. After the HEBS glass mask is written, the 

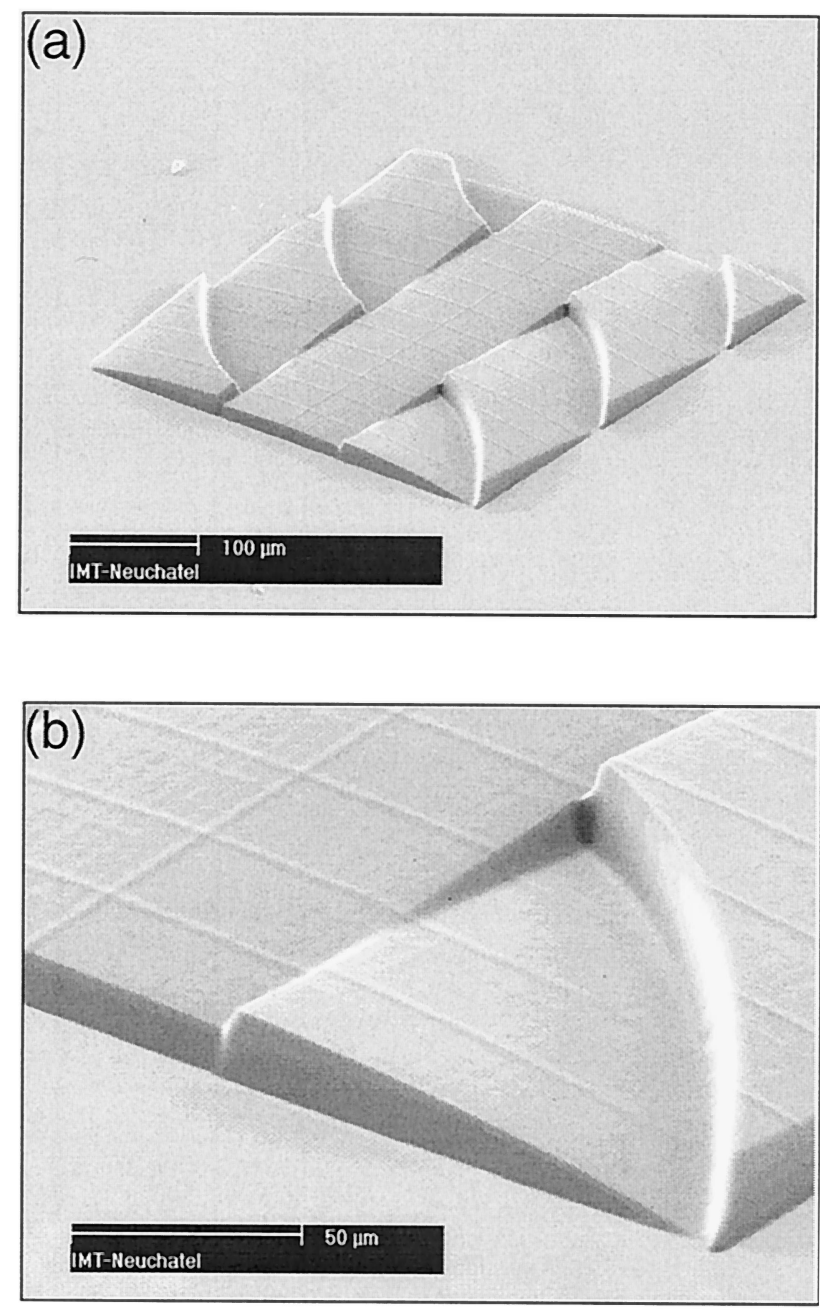

Fig. 7. Scanning-electron microscope image of the first element fabricated by HEBS glass gray-tone lithography in photoresist: (a) overview, (b) enlarged detail.

continuous surface-relief profiles are fabricated in a single lithography step. This technology is well adapted for the fabrication of deep micro-optical elements.

\section{B. Efficiency and Deflection-Angle Measurements}

Both types of micro-optical elements were fabricated by use of the three technologies. The laserwritten elements are in photoresist and were realized as continuous diffractive surface-relief profiles with a depth of $2.8 \mu \mathrm{m}$, designed for the second order (Fig. 4). Because the HEBS glass gray-tone technology (as well as the laser writing technique) offers the possibility of fabricating continuous surface-relief elements with a large profile depth $(15-20 \mu \mathrm{m})$, the elements can also be realized with many fewer zone transitions. In the example described here, the elements were realized as a continuous refractive surface profile with a depth of 18 $\mu \mathrm{m}$.

The elements fabricated by use of gray-tone lithog-
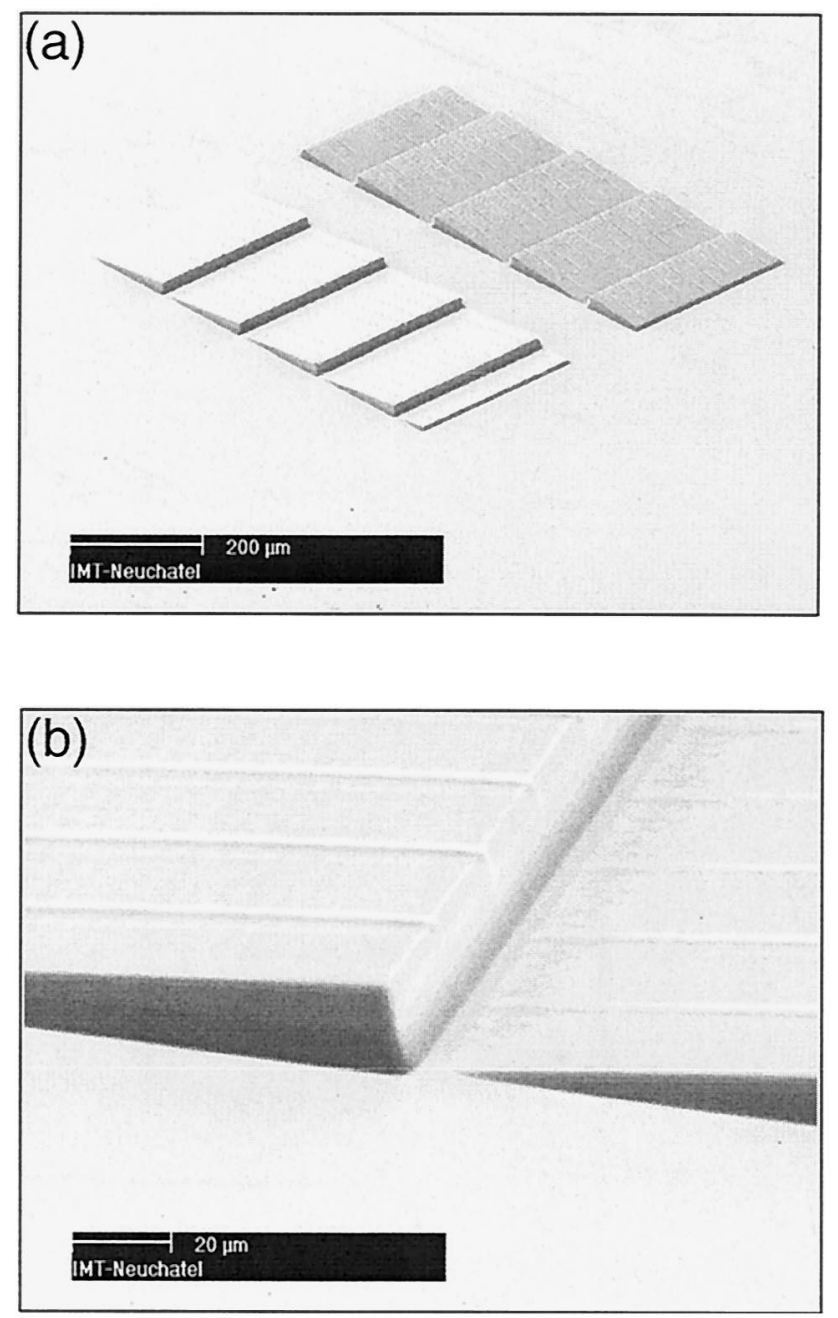

Fig. 8. Scanning-electron microscope image of the second element fabricated by HEBS glass gray-tone lithography in photoresist: (a) overview, (b) enlarged detail.

raphy in photoresist are shown in Figs. 7 and 8. The eight-level fused-silica elements have a diffractive surface profile with a depth of $1.65 \mu \mathrm{m}$. As a consequence of the relatively small grating periods of the first element compared with the minimum feature size of $1.25 \mu \mathrm{m}$, the effective number of phase levels per grating period was, on some parts of the element, less than 8. The first element fabricated by binary mask technology as an eight-level element in fused silica is shown in Fig. 9. One can clearly see the three parts of each of the elements with the different optical functions implemented.

Two main characteristics are of importance for the performance of the two DOEs in the beam-shaping device: the accuracy of the deflection angles at which the light deviates and the efficiency of the deflection into this direction. Therefore we analyzed the efficiency and the deflection angles of the elements fabricated by the three technologies. Table 3 shows a comparison of the measured diffraction efficiencies that were achieved with the different technologies. The efficiencies were measured with the 

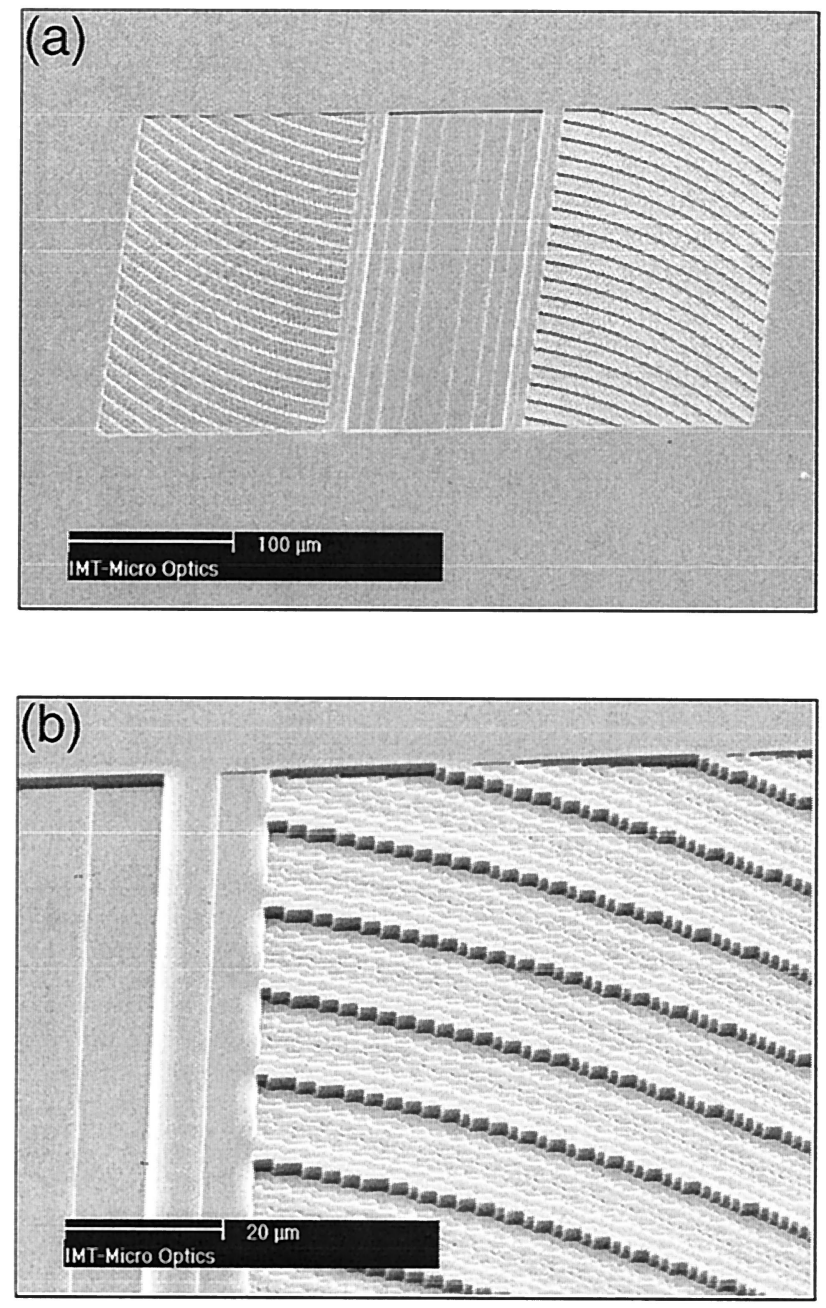

Fig. 9. Scanning-electron microscope image of the first element fabricated as a multilevel structure (eight levels) in fused silica: (a) overview, (b) enlarged detail.

focused light from a vertical-cavity surface-emitting laser diode at the design wavelength of $\lambda=850 \mathrm{~nm}$. The spot size (full width at $1 / e^{2}$-intensity level) in the plane of the element was measured with a knife-edge to be $50 \mu \mathrm{m}$. For the gray-tone elements the lesser of the two efficiencies includes the losses at the nonideal profile steps, whereas the higher efficiency (purely refractive) is measured when the beam does not hit such a step. We found that the efficiencies of the second element achieved with the three different technologies were nearly equal, slightly greater than $80 \%$. For the first element, which has steeper slopes
Table 4. Comparison of Measured and Designed Deflection Angles for the Two DOEs

\begin{tabular}{ccc}
\hline & $\begin{array}{c}\text { First Element } \\
\text { Deflection Angle, } \\
\text { Vertical }\end{array}$ & $\begin{array}{c}\text { Second Element } \\
\text { Deflection Angle }\end{array}$ \\
\hline $\begin{array}{c}\text { Pesign value } \\
\text { (deg) }\end{array}$ & 4.01 & 3.38 \\
$\begin{array}{c}\text { Multilevel (deg) } \\
\text { Laser written } \\
\quad \text { (deg) }\end{array}$ & 3.97 & 3.36 \\
Gray tone (deg) & 4.11 & 3.47 \\
\hline
\end{tabular}

and correspondingly smaller grating periods, the gray-tone element had a higher efficiency, whereas the laser-written elements and the multilevel elements performed nearly equally well. The sources of loss for the different elements are quite different. For the multilevel elements the losses are due mainly to the approximation of the ideal profile by the multilevel structure and to the alignment errors of the different lithographic steps. For the laser-written elements the main losses are caused by the finite width of the writing beam and surface roughness. The main losses of the gray-tone elements are due to surface roughness and to the nonideal profile steps.

Table 4 shows a comparison of the measured and the designed deflection angles of the two elements that were fabricated by the different technologies. The multilevel elements reproduced nearly exactly the designed deflection angles, the laser-written elements showed slight deviations from the design values, and the differences were largest for the gray-tone elements. For the multilevel elements the directions are determined by the grating periods, which are intrinsically well defined. For the refractive gray-tone elements the directions are determined by the surface profile, which is more difficult to control for the deep elements studied here.

For the diffractive elements, as fabricated by laser writing and multiple-projection lithography, we translated the difference between the measured and the designed deflection angles into the corresponding grating-period deviation. For the multilevel elements the corresponding grating-period deviation was approximately 60 and $90 \mathrm{~nm}$ for elements $\mathrm{A}$ and $\mathrm{B}$, respectively, which constitutes $5 \%-7 \%$ of the minimum feature size and a reasonable value for the accuracy of the $e$-beam writing of the chromium mask. For the laser-written ele-

Table 3. Measured Diffraction Efficiencies Normalized with Respect to Transmitted Intensity through an Unstructured Substrate

\begin{tabular}{llll}
\hline \multirow{2}{*}{$\begin{array}{c}\text { Type of } \\
\text { Efficiency }\end{array}$} & \multicolumn{2}{c}{ First Element (\%) } & Second Element (\%) \\
\cline { 2 - 4 } & \multicolumn{1}{c}{ Left Part } & Right Part & 82 \\
Multilevel & 70 & 68 & 81 \\
Laser writer & 78 (including steps) & 81 (including steps) & 78 (including steps) \\
Gray tone & 84 (purely refractive) & 84 (purely refractive) & 88 (purely refractive) \\
\hline
\end{tabular}


ments, we found corresponding grating-period deviations of approximately 220 and $280 \mathrm{~nm}$ for elements $\mathrm{A}$ and $\mathrm{B}$, respectively. The pixel size used for the raster scan during exposure was 400 $\mathrm{nm}$, and the grating-period deviations therefore correspond to approximately half of the pixel size, which is also a reasonable value.

\section{Conclusions}

We have designed, fabricated, and characterized a micro-optical incoherent beam-shaping device that is intended to optimize the coupling of a linear highpower diode laser into a multimode fiber. We achieved an overall efficiency of $28 \%$ with a first prototype, which corresponds well to the theoretical values obtained with a wave-propagation model. Straightforward improvements, such as antireflective coatings and the use of gray-tone elements, should lead to an efficiency of approximately $50 \%$. The beam-shaping device is compact, and the method of fabrication is well suited for mass production at low cost. Used in a range-finder measurement system, this micro-optical device will extend the measurement range because of the symmetrized intensity distribution obtained on a distant target.

We compared three technologies for the fabrication of the two micro-optical elements of the beamshaping device. Refractive-type elements (gray tone) yield better efficiency for large deflection angles, whereas diffractive elements (multilevel or laser written) give intrinsically accurate deflection angles.

This research was supported by the Swiss Priority Program Optique II. The authors thank the Centre Suisse d'Electronique et Microtechnique, Neuchâtel, Switzerland, for fabrication of the multilevel gratings in fused silica and E.-B. Kley, Friedrich-SchillerUniversität, Jena, Germany, for $e$-beam writing of the HEBS glass mask. We also thank Irene Philipoussis, Institute of Microtechnology, Neuchâtel, Christian Ossmann and Stefan Seider, Karl Suess AG, Munich, and Michael Scheidt for technical help.

\section{References}

1. B. Gächter, A. Schilling, L. Stauffer, U. Vokinger, "Optischer Entfernungsmesser," European patent application 00108836.8 (20 June 2000); patent pending.

2. J. R. Leger and W. C. Goltsos, "Geometrical transformation of linear diode-laser arrays for longitudinal pumping of solid state lasers," IEEE J. Quantum Electron. 28, 1088-1100 (1992).

3. J. N. Mait, "Understanding diffractive optic design in the scalar domain," J. Opt. Soc. Am. A 12, 2145-2158 (1995).

4. C. Kopp, L. Ravel, and P. Meyrueis, "Efficient beamshaper homogenizer design combining diffractive optical elements, microlens array and random phase plate," J. Opt. A 1, 398403 (1999).

5. P. Ehbets, H. P. Herzig, R. Dändliker, P. Regnault, and I. Kjelberg, "Beam shaping of high-power laser diode arrays by continuous surface-relief elements," J. Mod. Opt. 40, 637-645 (1993).

6. J. W. Goodmann, Introduction to Fourier Optics (McGraw-Hill, New York, 1996).

7. U. Vokinger, "Propagation, modification and analysis of partial coherent light fields," Ph.D. dissertation (Université de Neuchâtel, Neuchâtel, Switzerland, 1999).

8. M. T. Gale and M. Rossi, "Continuous-relief diffractive lenses and microlens arrays," in Diffractive Optics for Industrial and Commercial Applications, J. Turunen and F. Wyrowski, eds. (Akademie-Verlag, Berlin, 1997), Chap. 4.

9. M. T. Gale, Th. Hessler, R. E. Kunz, and H. Teichmann, "Fabrication of continuous-relief micro-optics: progress in laser writing and replication technology," in Diffractive Optics and Microoptics, Vol. 5 of 1996 OSA Technical Digest Series (Optical Society of America, Washington, D.C., 1996), p. 335.

10. M. B. Stern, "Binary optics fabrication" in Micro-Optics: Elements, Systems, and Applications, H. P. Herzig, ed. (Taylor \& Francis, London, 1997), Chap. 3.

11. C. Wu, "Method of making high energy beam sensitive glass," U.S. patent 5,078,771 (7 January 1992).

12. W. Däschner, C. Wu, and S. H. Lee, "General aspheric refractive micro-optics fabricated by optical lithography using a high energy beam sensitive glass gray-level mask," J. Vac. Sci. Technol. B 14, 135-138 (1996).

13. Ch. Gimkiewicz, D. Hagedorn, J. Jahns, E.-B. Kley, and F. Thoma, "Fabrication of microprisms for planar optical interconnections by use of analog gray-scale lithography with highenergy-beam-sensitive glass," Appl. Opt. 38, 2986-2990 (1999). 